\title{
DIMINISHING THE EARLINESS AND TARDINESS OF AGV USING PSBCSP ALGORITHM
}

\author{
MAHALAKSHMI ${ }^{1}$, SWATHI PAI $\mathbf{M}^{2}$, R MURUGESAN ${ }^{3}$ \\ ${ }^{1,3}$ School of Applied Sciences, REVA University, Bengaluru, Karnataka, India \\ ${ }^{2}$ Schoolof Computer Science and Applications, REVA University, Bengaluru, Karnataka, India
}

\begin{abstract}
As the earliness of the AGVs results in waiting and tardiness of AGVs results in temporary storage of the products in the shop floor, it is essential to minimize the earliness and tardiness of AGVs. Therefore we are proposing a mathematical optimization program in order to minimize the total earliness and total tardiness of AGVs in the manufacturing system. Since it is difficult to solve the mathematical program by conventional method, an optimization technique namely Positive Selection Based Clonal Selection Principle algorithm is used to obtain the optimal solution. The proposed algorithm is tested with the numerical examples and also compared with the other methods in the literature.

KEYWORDS: Flexible Manufacturing System, Automated Guided Vehicles, Earliness/Tardiness, Positive Selection Based Clonal Selection Principle
\end{abstract}

Received: Jun 08, 2020; Accepted: Jun 28, 2020; Published: Sep 18, 2020; Paper Id.: IJMPERDJUN20201334

\section{INTRODUCTION}

Moving semi-finished products from one shop to another shop and fully finished products from shops to ware house may be done by using cranes trucks, employees etc., which causes increase in the cost function of the manufacturing process. The new trend is to use Automated Guided Vehicles (AGVs), which increases flexibility and efficiency in the manufacturing process by reducing the cost function and make span. Manufacturing systems containing the AGVs are called as Flexible Manufacturing Systems (FMS). AGVs also plays a vital role in warehousing systems, container terminals, service industries and hospital managements. AGVs are material handling machineries, which travels on the network of guided path with a constant speed. These AGVs can travel in both forward and backward positions. Since simultaneously many vehicles are travelling in the same guided path, the collisions have to be avoided. A general review of AGVs are given in the literature [1, 2, and 3]. The recent works on AGVs scheduling and routing problems is given in the survey of [4]. An extensive review on operational control of AGVs are addressed in [5]. The conflict free route of AGV in a directional network is found by [6], which is based on finding the shortest path using the concept of time window graph. The petri - net model is also used to find the conflict free routing of AGVs [7 and 8]. An intelligent framework for conflict free shortest time path is presented by [9]. A mathematical program for routing problem of AGVs using Lagrangian decomposition techniques is provided in [10]. The routing in a mesh topologies and conflict free scheduling is given by [11]. A heuristic method of two stage traffic control scheme of AGVs is given by [12]. The minimization of make span using an optimization approach by assuming assignment tasks are given to AGVs is done in [13]. The dispatching and routing problems using heuristic method separately by performing scheduling first and in the next step the sequential path generation (SPG) is done by [14]. The combined problem of dispatching and also the conflict free 
routing of AGVs with two vehicles is proposed in [15]. An extension this work with four vehicles was proposed by [16]. The conflict free routing using ant colony algorithm is given by [17].By the above literature survey, it is found that there is gap of finding routing of AGVs along with minimization of Earliness and tardiness using Positive Selection Based Clonal Selection Principle (PSBCSP) algorithm method.Therefore in this paper a two stage Positive Selection Based Clonal Selection Principle (PSBCSP) algorithm is used in order to minimize the earliness and tardiness of AGV. Positive selection algorithm is developed by Seiden and Celeda in 1992 [18]. The main function positive selection algorithm [19] is to distinguish between the good and solutions in order to retain the good solution for future step and discard the bad solution permanently from the system. And the main objective of clonal selection principle [20, 21] is to select the best antibody for cloning and mutation in order to destroy the antigens. There two types of cells namely B-cells and T-cells for clonal selection principle. B-cell based clonal selection algorithm plays a vital role in solving various scheduling problems [22, 23, 24 and 25]. Both positive selection and clonal selection principle are combined to get a positive selection based clonal selection principle algorithm [26].

\section{MATHEMATICAL MODEL}

To study the proposed algorithm, we consider a job shop environment problem of multiple AGVs for the material handling. Sometimes the AGV guided path may be busy at the time of sending an AGV for material handling. Therefore, we need to obtain a free path for AGV movements. In the manufacturing process, the processing time of all jobs are planned clearly. If an AGV reports early to the shop floor, it has to wait until the processing is finished. This waiting time is regards with the due date of the jobs. This may delay the delivery of the product outlets in the manufacturing process. An essential driver of the manufacturing industry is to deliver the products in the correct time. Therefore, in this paper we are computing the manufacturing schedule of $\mathrm{AGVs}$ along with routing in order to minimize the total earliness/ total tardiness of AGVs in the job shop environment. The following assumptions are made before modelling the problem

- Number of jobs, number of AGVs and the number of shops are given earlier.

- Processing time of each job is specified clearly.

- At a time, an AGV can transport only a single load.

- The velocities of all AGVs are same

The mathematical formulation for the proposed algorithm is given as follows

Indices

$i \quad$ Number of jobs, $i=1,2 \ldots m$

$j \quad$ Number of AGVs, $j=1,2 \ldots n$

$s, s^{\prime} \quad$ Number of Shops, $s=1,2 \ldots . . o$ and $s^{\prime}=2,3 \ldots . o$

\section{Parameters}

$W T_{e} \quad$ Weight of total earliness of the job, $W T_{e} \geq 0$

$W T_{d} \quad$ Weight of total tardiness of the job, $W T_{d} \geq 0$

$P_{i s} \quad$ Processing time of the job $i$ processed in the shop $s$ 


$$
\begin{array}{ll}
d u_{i s} & \text { Due date for job } i \text { being processed in shop } s \\
d_{s s^{\prime}} & \text { Distance between the shops } s \text { and } s^{\prime} \\
V_{e} & \text { Velocity of the AGV (equal for all AGVs) } \\
t_{j s s^{\prime}} & \text { Travelling time for an AGV } j \text { between the shops } s \text { and } s^{\prime} \\
E_{i s} & \text { Earliness of the job } i \text { processed in shop } s \\
T_{i s} & \text { Tardiness of the job } i \text { processed in shop } s \\
C_{i s} & \text { Completion time of job } i \text { in shop } s \\
A_{i s} & \text { Allocation of job } i \text { to shop } s \\
A_{i s}=\left\{\begin{array}{c}
1 \text { if job } i \text { is allocated to shop } s \\
0 \text { otherwise }
\end{array}\right.
\end{array}
$$

\section{Decision Variables}

$$
\begin{gathered}
x_{i j s}=\left\{\begin{array}{r}
1 \text { if job } i \text { is assigned to AGV } j \text { in shop } s \\
0 \text { otherwise }
\end{array}\right. \\
y_{j s s},=\left\{\begin{array}{c}
1 \text { if an AGV } j \text { moves between the shops } s \text { and } s \\
0 \text { otherwise }
\end{array}\right.
\end{gathered}
$$

\section{Objective Functions}

The objective function is to minimize

$$
z=\sum_{s=1}^{o} \sum_{i=1}^{m}\left(W T_{e} \cdot E_{i s}+W T_{d} \cdot T_{i s}\right)
$$

(1)

Where

$$
W T_{e}=\sum_{s=1}^{o} \sum_{i=1}^{m}\left|P_{i s}-d u_{i s}\right|^{2} \text { and } W T_{d}=\sum_{s=1}^{o} \sum_{i=1}^{m}\left|P_{i s}-C_{i s}\right|^{2}
$$

(2)

\section{Constraints}

- $\quad$ For each $s^{\text {th }}$ shop exactly one AGV is scheduled

$$
\sum_{i=1}^{m} \sum_{j=1}^{n} \sum_{s=1}^{o} x_{i j s}=1
$$

(3)

- If an AGV is assigned to one shop, then the path between that shop and the immediate next shop is busy

$$
x_{i j s} \cdot y_{j s s},=1
$$


(4)

Where $i=1,2 \ldots m, j=1,2 \ldots n, s=1,2 \ldots . . o$ and $s^{\prime}=2,3 \ldots . o$

- $\quad$ Only one AGV is assigned to each shop

$$
\sum_{j=1}^{n} \sum_{s=1}^{o} \sum_{s^{\prime}=2}^{o} y_{j s s^{\prime}}=1
$$

(5)

- In a job shop allocation only one AGV is moved in the path between any two nodes

$$
\sum_{i=1}^{m} \sum_{j=1}^{n} \sum_{s=1}^{o} y_{j s s^{\prime}} \cdot A_{i s}=1, s^{\prime}=s+1
$$

(6)

- $\quad$ In job shop allocation only one AGV is assigned

$$
\sum_{i=1}^{m} \sum_{j=1}^{n} \sum_{s=1}^{o} x_{i j s} \cdot A_{i s}=1, s^{\prime}=s+1
$$

(7)

- The processing times of the jobs in the shops is given by

$$
P_{i s}=\sum_{i=1}^{m} \sum_{j=1}^{n} \sum_{s=1}^{o} \sum_{s^{\prime}=2}^{o} x_{i j s} \cdot t_{j s s^{\prime}}
$$

- The relationship between completion time of the job in previous shop and processing time of the job in the next shop is given by

$$
C_{i s} \geq C_{i s-1}+P_{i s}, i=1,2 \ldots m, s=1,2 \ldots \ldots
$$

(9)

- $\quad$ The tardiness of the job is given by

$$
T_{i s}=\max \left\{t_{j s s^{\prime}}, C_{i s}-d u_{i s}\right\}
$$

(10) Where $i=1,2 \ldots m, j=1,2 \ldots n, s=1,2 \ldots . . o$ and $s^{\prime}=2,3 \ldots .$.

- The Earliness of the jobs is given by

$$
E_{i s}=\max \left\{t_{j s s^{\prime}}, d u_{i s}-C_{i s}\right\}
$$

(11)

Where $i=1,2 \ldots m, j=1,2 \ldots n, s=1,2 \ldots .$. and $s^{\prime}=2,3 \ldots . . o$ 


\section{IMPLEMENTATION OF POSITIVE SELECTION BASED CLONAL SELECTION PRINCIPLE}

We have taken test problem to study the proposed algorithm which is given in the table 1

Table 1: Test problem for proposed algorithm

\begin{tabular}{|l|c|}
\hline Parameters & Values \\
\hline Number of jobs & 45 \\
\hline Number of AGVs & 12 \\
\hline Number of shops & 17 \\
\hline
\end{tabular}

The method is explained in two stages. In the first stage positive selection algorithm is used and in the second stage clonal selection principle is applied.

\section{Positive Selection Algorithm}

Upon the generation of the initial solution to the problem, the main function of positive selection principle algorithm is to distinguish between the good and bad solution in order to discard bad solution from the system. In the human body, good solutions represents the antibodies which recognizes and overcome the antigen. The following figure 1 represents the working rule of positive selection principle in the human body.

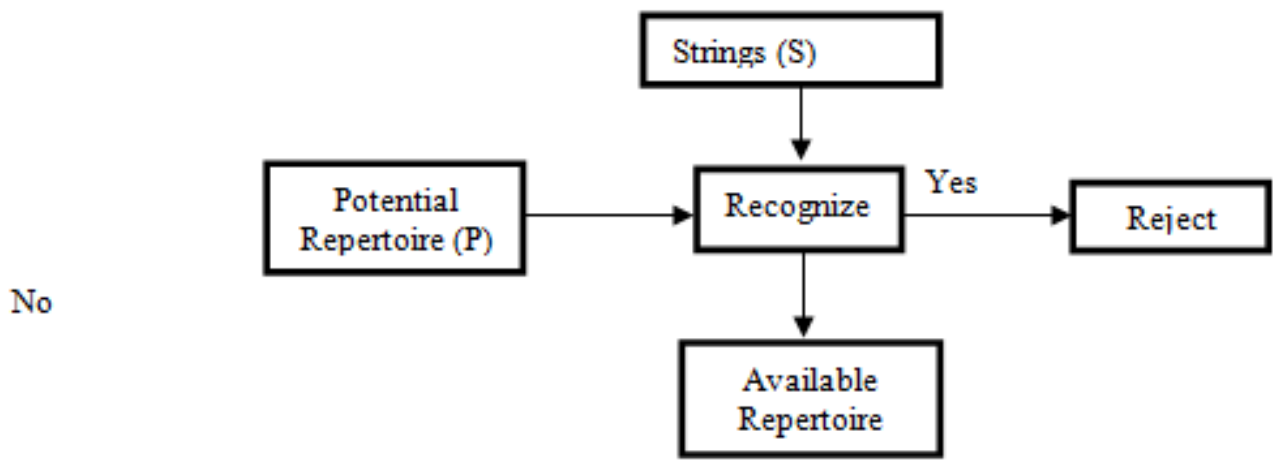

Figure1: Positive Selection Algorithm

Consider a case of infection (viral, bacterial) attacking the human body. The string $\mathrm{S}$ is the set of antigens attacking the human body which has to be destroyed. Potential Repertoire $(\mathrm{P})$ represents the potentially T-cells in human immune system. To destroy the antigens, we need the cells which can overcome the antigen. Therefore all cells in the human body are tested to find whether they recognize the antigen or not. The cells which possess capacity of recognition of the antigens are called Available Repertoire (A), which will be retained for further usage of destroying the antigens. The remaining cells which fails in the recognition of antigens are discarded from the system. The positive selection algorithm is applied for filtering purposes. Those solutions satisfying certain criteria is retained, other solutions are completely discarded from the system. To check the satisfaction level of the solution, we are fixing a threshold value. A solution is retained if the optimal value of the solution is less than the threshold, otherwise it is completely discarded from the system

\section{Clonal Selection Algorithm}

The clonal selection algorithm is based on the working principle of human immune system. B-cells along with the T-cells will destroy the various viruses and bacteria which attacks the human body. But it has been observed that B-cells has 
unique tendency to clone those cells which are capable of destroying antigens attacking the human body. Upon the attack of antigen a particular string formation of B-cells are done to overcome the infection. The cell will clone for a large amount to produce high capacity antibodies to overcome the antigen. Usually B-cells themselves under goes process of cloning and mutation repeatedly to recognize the right solution to destroy the antigens. The flow for the positive selection based clonal selection principle algorithm is given in the figure 2.

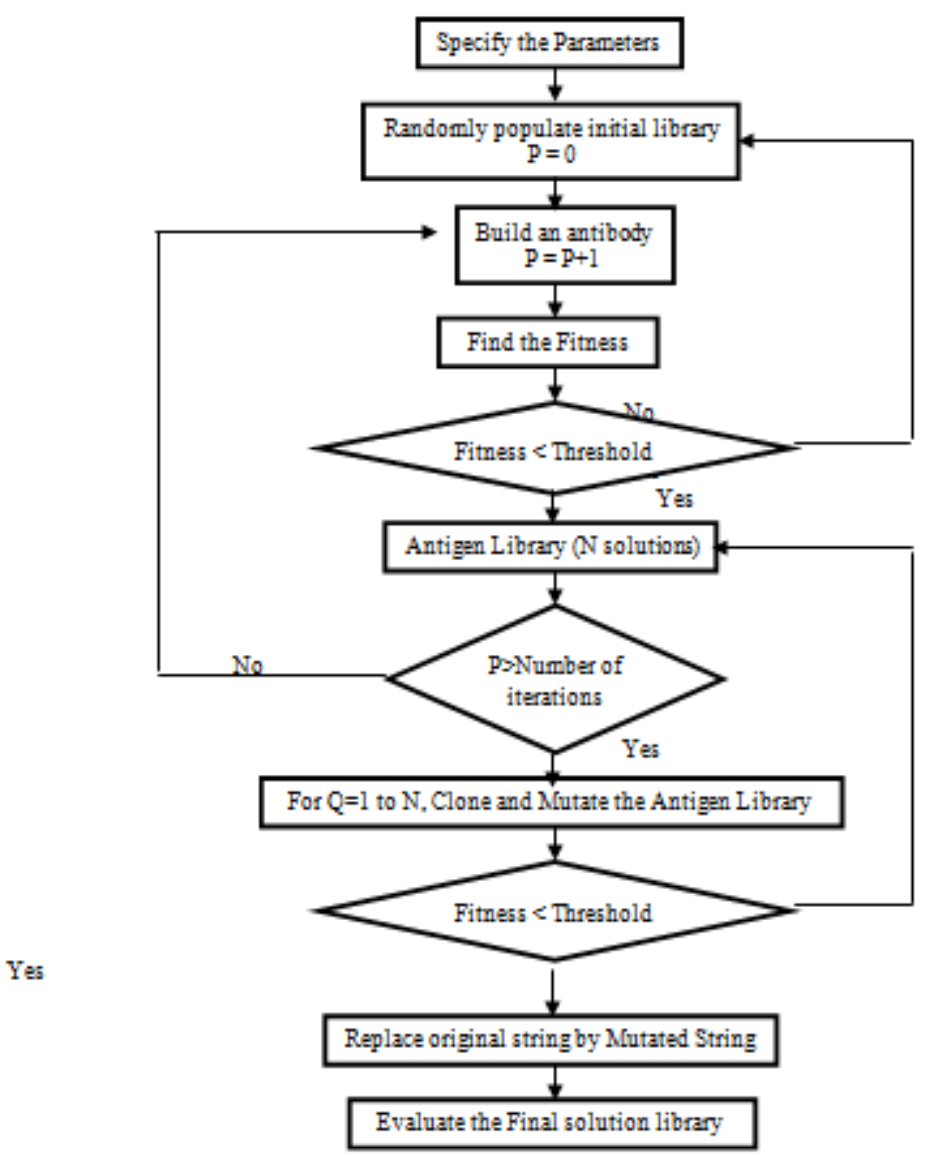

Figure 2: Flow chart of the proposed Algorithm

\section{RESULTS AND DISCUSSIONS}

To study performance of the proposed algorithm, it must be simulated in MATLAB R2012a (7.14.0.739), 64-bit (win64). The entire tests were executed in Intel i5 processor under the Microsoft windows 7(64-bit) operating system. The obtained variables are given in the table 2 and a comparison analysis of the proposed algorithm with the other existing algorithm is given in the table 3 as well as in figure 2.

Table 2: Variable of the solution

\begin{tabular}{c}
$x_{312} x_{1191} x_{1345} x_{1536} x_{3187} x_{25610} y_{234} y_{3410} y_{2212} y_{5133} y_{3102} y_{71013}$ \\
$x_{2673} x_{1264} x_{22112} x_{3177} x_{1728} x_{1910} y_{457} y_{10112} y_{2113} y_{677} y_{81714} y_{71614}$ \\
$x_{4415} x_{526} x_{1085} x_{24126} x_{23611} x_{36111} y_{1124} y_{1233} y_{3211} y_{8103} y_{12174} y_{9812}$ \\
$x_{7314} x_{21813} x_{35916} x_{3719} x_{341012} x_{20210} y_{4138} y_{821} y_{1117} y_{6817} y_{5911} y_{6165}$ \\
\hline
\end{tabular}


$x_{9102} x_{6107} x_{1438} x_{16118} x_{41916} x_{38122} y_{1169} y_{1410} y_{3159} y_{8610} y_{9154} y_{16126}$

$x_{29114} x_{32715} x_{42210} x_{4511} x_{4553}-y_{9128} y_{1178} y_{1297} y_{1076} y_{1111}-$

$x_{12123} x_{8613} x_{28517} x_{4044} x_{2739}-y_{456} y_{4119} y_{5616} y_{6141} y_{51516}-$

$x_{2051} x_{301214} x_{39119} x_{4445} x_{18517}-y_{2135} y_{732} y_{1815} y_{7145} y_{81015}-$

Table 3: Comparison table of the proposed algorithm

\begin{tabular}{|c|c|c|c|c|}
\hline Methods & Approaches & $\begin{array}{c}\text { Size of the } \\
\text { problem }\end{array}$ & $\begin{array}{c}\text { Optimal } \\
\text { Value }\end{array}$ & Run time(min) \\
\hline \multirow{3}{*}{$\begin{array}{l}\text { Gerstl and } \\
\text { Mosheiov [23] }\end{array}$} & \multirow{3}{*}{ Agent based } & $10 * 5 * 5$ & 2327 & 145 \\
\hline & & $20 * 10 * 15$ & NFS & 6000 \\
\hline & & $45 * 12 * 17$ & NFS & 6000 \\
\hline \multirow{3}{*}{$\begin{array}{l}\text { Tanaka and Araki } \\
\text { [24] }\end{array}$} & \multirow{3}{*}{ Lagrangian relaxation } & $10 * 5 * 5$ & 2401 & 77 \\
\hline & & $20 * 10 * 15$ & 6417 & 3401 \\
\hline & & $45 * 12 * 17$ & NFS & 6000 \\
\hline \multirow{3}{*}{$\begin{array}{l}\text { Kianfar and } \\
\text { Moshlehi [25] }\end{array}$} & \multirow{3}{*}{ Branch and bround } & $10 * 5 * 5$ & 2278 & 66 \\
\hline & & $20 * 10 * 15$ & 6532 & 2987 \\
\hline & & $45 * 12 * 17$ & 91345 & 5467 \\
\hline \multirow{3}{*}{ Janiak et al. [26] } & \multirow{3}{*}{ Dynamic programming } & $10 * 5 * 5$ & 2205 & 43 \\
\hline & & $20 * 10 * 15$ & 6347 & 197 \\
\hline & & $45 * 12 * 17$ & 8765 & 4986 \\
\hline \multirow{3}{*}{$\begin{array}{l}\text { Elyashi and } \\
\text { Salmasi [27] }\end{array}$} & \multirow{3}{*}{$\begin{array}{l}\text { Chance constrained } \\
\text { programming }\end{array}$} & $10 * 5 * 5$ & 2491 & 54 \\
\hline & & $20 * 10 * 15$ & 6691 & 81 \\
\hline & & $45 * 12 * 17$ & NFS & 6000 \\
\hline \multirow{3}{*}{$\begin{array}{l}\text { Droubouchevitch } \\
\text { and Sidney [28] }\end{array}$} & \multirow{3}{*}{$\begin{array}{l}\text { Two stage heuristic } \\
\text { algorithm }\end{array}$} & $10 * 5 * 5$ & 2709 & 238 \\
\hline & & $20 * 10 * 15$ & 6987 & 4671 \\
\hline & & $45 * 12 * 17$ & NFS & 6000 \\
\hline \multirow{3}{*}{$\begin{array}{l}\text { Hamed and } \\
\text { Mohammad [29] }\end{array}$} & \multirow{3}{*}{ Heuristics } & $10 * 5 * 5$ & 2114 & Negligible \\
\hline & & $20 * 10 * 15$ & 6323 & Negligible \\
\hline & & $45 * 12 * 17$ & 75438 & Negligible \\
\hline \multirow{3}{*}{ Proposed algorithm } & \multirow{3}{*}{$\begin{array}{l}\text { Positive Selection Based } \\
\text { Clonal Selection } \\
\text { Principle(PSBCSP) }\end{array}$} & $10 * 5 * 5$ & 2004 & Negligible \\
\hline & & $20 * 10 * 15$ & 6022 & Negligible \\
\hline & & $45 * 12 * 17$ & 64565 & Negligible \\
\hline
\end{tabular}




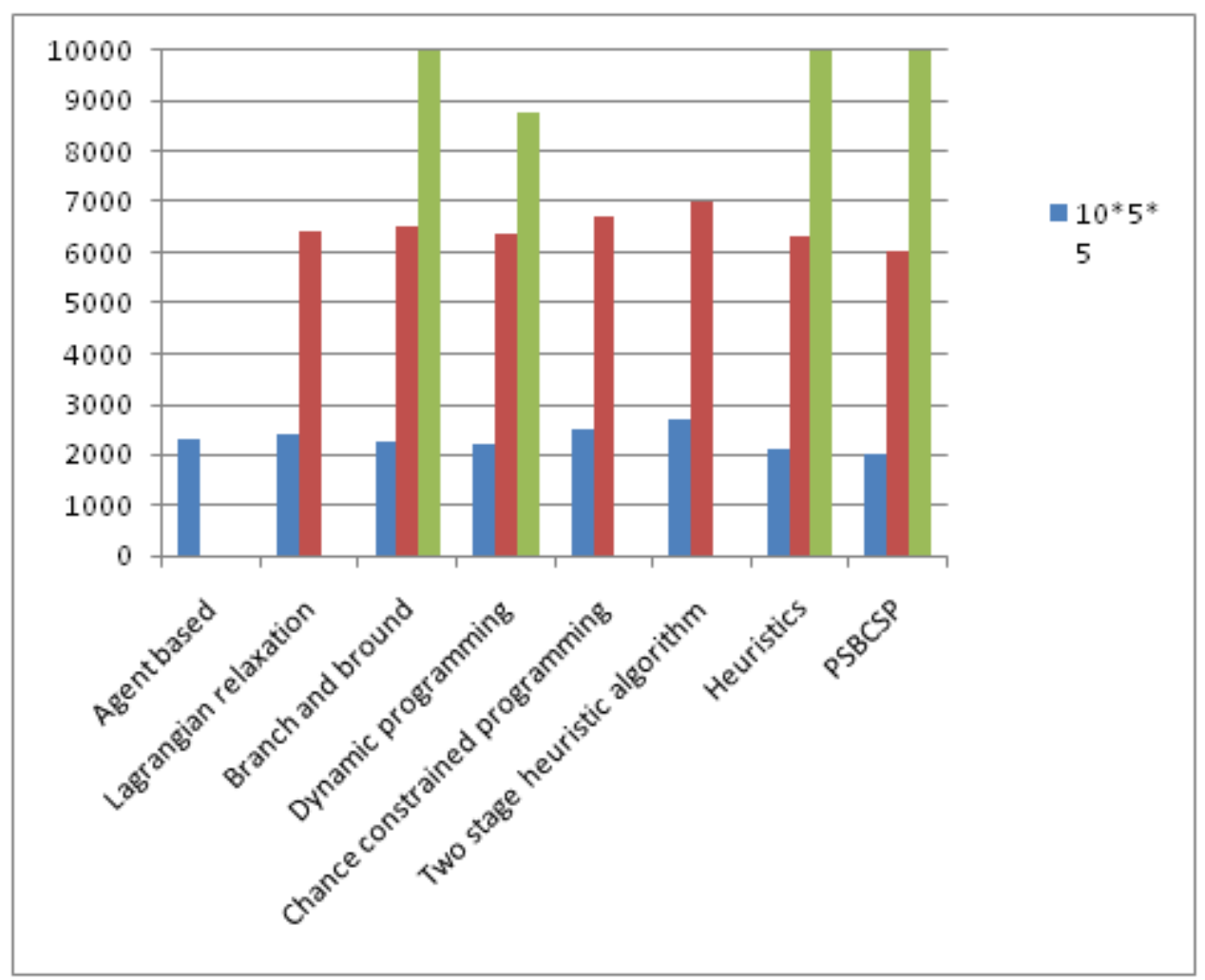

Figure 2: Comparison of the proposed algorithm with other algorithm

\section{CONCLUSIONS}

An essential driver to the manufacturing industries is to deliver the products on time to the customer. This is possible only if we minimize the make span of the manufacturing process. As a part of it, in this paper we are minimizing the total earliness and tardiness of AGVs to reduce the make span. The proposed algorithm is modelled by assuming number of jobs, number of AGVs, number of shops and processing time of each jobs are given. Along with that it is assumed that all AGVs moves with the same speed and each AGV can carry a unit load at a time. Scheduling, conflict free routes and optimal values are found using proposed algorithm. The obtained result is compared with the other existing models in the literature. This shows that the proposed algorithm yields a better result compared to others. We can solve the same modelusingotherhybridalgorithms.

\section{REFERENCES}

1. C.G. Co, J.M.A. Tanchoco, A review of research on AGVs vehicle management, Eng. Costs Prod. Econ. 21 (1991) 35-42.

2. R.E. King, C.A. Wilson, A review of automated guided vehicle system design and scheduling, Prod. Plan. Control 2 (1991) $44-$ 51.

3. T. Ganesharajah, N.G. Hall, C. Sriskandarajah, Design and operational issues in AGV-served manufacturing systems, Ann. Oper. Res. 76 (1998) 109-154.

4. L. Qiu, W.-J. Hsu, H. Wang, Scheduling and routing algorithms for AGVs: a Survey, Int. J. Prod. Res. 40 (2002) $745-$ 760.I.F.A. Vis, Survey of research in the design and control of automated guided vehicle systems, European J. Oper. Res. 170 (2006) 677-709.

5. I.F.A. Vis, Survey of research in the design and control of automated guided vehicle systems, European J. Oper. Res. 170 (2006) 677-709. 
6. C.W. Kim, J.M.A. Tanchoco, Conflict-free shortest time bi-directional AGV routing, Int. J. Prod. Res. 29 (1991) 2377-2391.

7. M. Dotoli, M.P. Fanti, Coloured timed Petri net model for real-time control of automated guided vehicle systems, Int. J. Prod. Res. 9 (2004) 1787-1814.

8. N. Wu, M. Zhou, Modeling and deadlock avoidance of automated manufacturing systems with multiple automated guided vehicles, IEEE Trans. Syst. Man Cybern. B 35 (2005) 1193-1201.

9. S.P. Singh, M.K. Tiwari, Intelligent agent framework to determine the optimal conflict-free path for an automated guided vehicle system, Int. J. Prod. Res. 40(2002) 4195-4223.

10. T. Nishi, M. Ando, M. Konishi, Distributed route planning for multiple mobile robots using an augmented Lagrangian decomposition technique, IEEE Trans. Robot. 22 (2005) 1191-1200.

11. S. Ghasemzadeh, E. Behrangi, M.A. Azgomi, Conflict-free scheduling and Routing of automated guided vehicles in mesh topologies, Robot. Auton. Syst.30 (2009) 738-748.

12. J.H. Lee, B.H. Lee, M.H. Choi, A real-time traffic control scheme of multiple AGV systems for collision free minimum time motion: a routing table approach, IEEE Trans. Syst. Man Cybern. A 28 (1998) 347-358.

13. N.N. Krishnamurthy, R. Batta, M.H. Karwan, Developing conflict-free routes for automated guided vehicles in a flexible manufacturing system, Oper. Res. 41(1993) 1077-1090.

14. C. Oboth, R. Batta, M. Karwan, Dynamic conflict-free routing of automated guided vehicles, Int. J. Prod. Res. 37 (1999) 2003-2030.

A. Langevin, D. Lauzon, D. Riopel, Dispatching, routing and scheduling of two automated guided vehicles in a flexible manufacturing system, Int. J. Flexible Manuf. Syst. 8 (1996) 246-262.

15. G. Desaulniers, A. Langevin, D. Riopel, B. Villeneuve, Dispatching and conflict free routing of automated guided vehicles: an exact approach, Int. J. Flexible Manuf. Syst. 15 (2003) 309-331.

16. Mohammad Saidi-Mehrabad, Saeed Dehnavi-Arani, Farshid Evazabadian, Vahid Mahmoodian. An Ant Colony Algorithm (ACA) for solving the new integrated model of job shop scheduling and conflict-free routing of AGVs, Computers \& Industrial Engineering. 86 (2015) 2-13.

17. Seiden P E and Celada F 1992 A Model for Simulating Cognate Recognition and Response in the Immune System Journal of Theoretical Biology 158 pp. 329 - 357

18. Van Truong Nguyen, Xuan Hoai Nguyen and Chi Mai Luong 2013 A Novel Combination of Negative and Positive Selection in Artificial Immune Systems IEEE RIVF Int Conf on Computing \& Communication Technologies - Research, Innovation, and Vision for the future (RIVF)

19. Murugesan R, Balan K.S and Kumar V N 2010 Clonal selection algorithm using improved initialization for solving JSSP Proc of IEEE Int Conf on Communication Control and Computing Technologies (ICCCCT) pp 470-475

20. Leandro N de Castro and Fernando J Von Zuben 2002 Learning and Optimization Using the Clonal Selection Principle ieee transactions on evolutionary computation vol. 6 no3

21. Carlos A Coello, Daniel Cortes Rivera and Nareli Cruz Cortes Use of an Artificial Immune System for Job Shop Scheduling In the Proceeding of Second International Conference on Artificial Immune Systems (ICARIS) September 1-3, 2003, Napier University, Edinburgh, UK. 
22. E Hart and P Ross 1999 The Evolution and Analysis of a potential Antibody Library for use in Job-Shop Scheduling A chapter in the book New Ideas in Optimization pp 185-202 McGraw-Hill

23. E Hart, Ross $P$ and Nelson J 1998 Producing robust schedules via an artificial immune system Int Conf on Evolutionary Computing Alaska USA pp 464-469 IEEE Press

24. F Celada and P E Seiden 1998 Modeling Immune Cognition Proc of the IEEE Int Conf on Systems Man and Cybernetics October 11-14

25. Murugesan.R 2012 Positive Selection Based Modified Clonal Selection Algorithm for Solving Job Shop Scheduling Problems Applied Mathematical Sciences Vol 6 2255-2271 